\title{
MÁS ALLÁ DE LA DOCENCIA: LA UNIDAD DE ATENCIÓN PSICOLÓGICA Y SALUD MENTAL DEL UNIVERSITARIO (UAPSMU) EN LA UNIVERSIDAD DE SALAMANCA (1996-2004)
}

\section{BEYOND TEACHING: THE UNIVERSITY MENTAL HEALTH AND PSYCHOLOGICAL ASSISTANCE UNIT (UAPSMU) AT UNIVERSITY OF SALAMANCA (1996-2004)}

\author{
Alejandro Ávila Espada ${ }^{1}$, Jesús R. Herrero SánCheZ² y Luz Célia Fernández \\ MARTÍN $^{3}$ \\ 1 Universidad Complutense. Catedrático de Psicoterapia. Dep. de Personalidad, Evaluación y Tratamiento \\ Psicológicos I (Psicología Clínica), Facultad de Psicología, Universidad Complutense de Madrid. \\ Catedrático de la Universidad de Salamanca 1991-2004. Director UAPSMU 1996-2004. Dirección de \\ contacto: avilaespada@psi.ucm.es \\ ${ }^{2}$ Universidad de Salamanca. Prof. Titular Dep. Personalidad, Evaluación y Tratamiento Psicológicos, \\ Universidad de Salamanca. Miembro UAPSMU desde 1996. \\ ${ }^{3}$ Universidad de Salamanca. Prof. Asociada, Dep. Personalidad, Evaluación y Tratamiento Psicológicos, \\ Universidad de Salamanca. Miembro UAPSMU desde 1996.
}

\section{Resumen}

La Atención Psicológica al Universitario se ofrece como un Servicio especializado en algunas Universidades españolas desde hace dos décadas. La Universidad de Salamanca está entre las pioneras en ofertar a la comunidad universitaria un servicio específico de atención psicológica y primera intervención en salud mental en su propio entorno, a un grupo poblacional definido principalmente por el desarraigo de su domicilio familiar, ya que una elevada proporción de su alumnado procede de otras comunidades, a veces muy distantes, así como por las características del propio tramo etáreo de los jóvenes universitarios, entre 18 y 25 años, un momento de eclosión, tanto de crisis vitales evolutivas, como de patologías graves. En este trabajo se ofrecen datos sobre la historia de esta Unidad en la Universidad de Salamanca, desde su puesta en marcha en el año 1996, hasta su reformulación en 2004. El servicio fue de carácter gratuito y exclusivo para todos los miembros de la comunidad universitaria. La atención fue desa-

\begin{abstract}
College Mental Health Counselling is a specialized service offered in several Spanish Universities some decades ago. Salamanca University is between the pioneers to offer university community a specialized service of Psychological Aid and First Intervention on Mental Health within their own campus, for a population group characterized by rootlessness of their family environment, usually far from their home environment, and with an age between 18 and 25, a period with life crises and severe pathological onsets. In this paper some data on the history of this Unit in Salamanca University is offered, from the beginning of the service in 1996 to their change in 2003. The service was free-cost, only for university people, and the professional staff included professors and professional psychologists under a scheme of non-profit collaborations. The main indicators of the interventions and people attended during this period are exposed, and also the main intervention programs developed:
\end{abstract}


rrollada mediante un equipo de psicólogos y profesores que colaboraron con carácter voluntario y gratuito. Se analiza la demanda atendida en este período, y se detallan los principales programas de intervención desarrollados: Enfrentamiento de la ansiedad ante los exámenes e Intervención ante la problemática del desarraigo, entre otros. Finalmente se hacen una serie de consideraciones sobre la organización y objetivos de este tipo de servicios.

\section{Palabras Clave}

Salud Mental en la Universidad, Ansiedad ante los Exámenes, Problemática del desarraigo familiar.

\section{Una referencia histórica}

La Unidad de Atención Psicológica y Salud Mental para el Universitario (UAPSMU) tuvo su origen en Febrero de 1996, en un Proyecto impulsado por el equipo rectoral de la Universidad $^{1}$, cuya coordinación asumió el Profesor Alejandro Ávila Espada y en el que participaron distintos Departamentos de la Facultad de Psicología y de otras Facultades de la Universidad de Salamanca ${ }^{2}$. Enmarcado en una tradición que contaba ya con experiencias en otras universidades españolas, conviene recordar los orí-
Facing Exams Anxiety; Care on rootlessness from family environment and attachment problems, between others. Finally we made some considerations on the structure and objectives for this type of services.

\section{Key Words}

College Mental Health Counselling, Exams Anxiety, Family rootlessness problems. genes de los Servicios de Asesoramiento, Consejo, Evaluación y Tratamiento Psicológicos, dependientes de las Universidades, que se encuentran en el ámbito anglosajón y germánico, desde donde se comenzaron a articular dichos programas como una necesidad para los estudiantes. Entre los antecedentes de iniciativas de este tipo en las Universidades españolas (véase la tabla 1), destacan las de Universidad Autónoma de Madrid (Servicio de Psicología Aplicada, Facultad de Psicología); U.N.E.D. (Unidad de Terapia de Conducta); Universidad Complutense (Unidad de Psicología Clínica y de la Salud); Universidad de Barcelona (Facultad de Psicolo-

1 La propuesta surgió del Vice-Rectorado de Asistencia al Universitario (Prof. D. Enrique Cabero Morán) y de su Servicio de Asistencia al Universitario (Prof. Teodoro Andrés López; posteriormente Prof. Santiago Rodríguez Vicente) quienes con su voluntad de servicio y entusiasmo hicieron posible la puesta en marcha del Servicio. La propuesta de diseño y puesta en marcha de la Unidad en la Facultad de Psicología la asumió el Profesor Alejandro Ávila Espada, Catedrático de Personalidad, Evaluación y Tratamiento Psicológicos, con el apoyo de su departamento y los Decanos que hubo en ese período: Ramón Fernández Pulido y José Luis Vega.

2 El Servicio se organiza principalmente en torno a un grupo de profesores del Departamento de Personalidad, Evaluación y Tratamiento Psicológicos, con colaboraciones de algunos profesores de otros departamentos de la Facultad de Psicología, y de los Departamentos de Psiquiatría y Trabajo Social. Entre los profesores que colaboraron de manera más continuada y a lo largo de todo el proyecto están (por orden alfabético, no es una lista exhaustiva) Alejandro Ávila Espada, Marta Badia Corbella, Ricardo Canal Bedia, Luz Celia Fernández Martín, Antonio García de la Hoz, María Teresa González Martínez, Jesus R. Herrero Sánchez, Cristina Jenaro Rio, Isabel Serrano Pintado, entre otros. Colaboraron también los entonces doctorandos, y ahora profesores en otras universidades: Luis Ángel Saúl Gutiérrez, Belén Gutiérrez Bermejo, Elena Felipe Castaño. Tuvieron también un papel destacado otros profesionales: José González Guerras, Esperanza Gómez Gazol, Janine J. Vidal, Yolanda Noelia Sánchez, M. ${ }^{\circ}$ Camino Escolar. 
Tabla 1. Creación de los Servicios de Atención Psicológica en las universidades españolas.

\begin{tabular}{|l|c|c|c|}
\hline \multicolumn{1}{|c|}{ Universidades } & $\begin{array}{c}\text { Año de inicio de } \\
\text { actividades }\end{array}$ & Coste & Usuarios \\
\hline Universidad de Barcelona & $1985-1995$ & $\begin{array}{c}1985 \text { Gratuito } \\
1995 \text { de Pago }\end{array}$ & SA \\
\hline Universidad Politécnica de Cataluña & 1991 & Gratuito & SC \\
\hline Universidad de Málaga & 1992 & Gratuito & SC \\
\hline Universidad de Almería & 1994 & Gratuito & SC \\
\hline Universidad Autónoma de Barcelona & 1995 & Gratuito & SC \\
\hline Universidad de Salamanca & 1996 & Gratuito & SC \\
\hline Universidad N. de Educación a Distancia & 1998 & de Pago & SA \\
\hline Universidad Complutense de Madrid & 1999 & Gratuito & SC \\
\hline Universidad Jaime I de Castellón & 1999 & de Pago & SA \\
\hline Universidad Ramón Llull de Barcelona & 1999 & Gratuito & SC \\
\hline
\end{tabular}

Nota: SA (Servicio Abierto a la Comunidad Universitaria y sociedad en general); SC (Servicio Cerrado, en exclusividad a los miembros de la Comunidad Universitaria). Adaptado de Clinical Psychology in Spain (p. 697), por V. del Barrio y H. Carpintero, 2003. Journal of Clinical Psychology, 59 (6), 687-699.

gía); Universidad de Valencia (Departamento de Personalidad, Evaluación y Tratamiento Psicológicos); Universidad de Sevilla (SOU); Universidad de Granada (Unidad de Clínica Conductual) y Universitat Ramon Llull (Barcelona), mientras que en otras muchas su proyecto se desarrolló posteriormente al inicio de nuestra experiencia, o está en fase avanzada. Todas ellas nos sirvieron de referente inicial en la construcción de un Proyecto que acaba teniendo una orientación y una idiosincrasia propia de nuestro ámbito espacio-temporal: La Universidad de Salamanca, dividida en cuatro campus, Salamanca, Zamora, Béjar y Ávila.

\section{Proyecto marco}

La Universidad de Salamanca ha estado entre las pioneras en España al crear un servicio asistencial y preventivo ofertado gratuitamente a la Comunidad Universitaria, articulado con la Red pública asistencial de Salud Mental y Servicios Sociales. Se trata de un desarrollo de sus competencias y objetivos de atención a la problemática general que afecta al estudiante en el marco de convivencia y formación que constituye la Universidad. Ya desde hace años, y en el marco del Servicio de Orientación se venían realizando colaboraciones de manera desinteresada por ciertos profesionales de la Universidad afines a estas problemáticas, aunque a título individual y sin una coordinación o planes de acción específicos. Para facilitar dicha coordinación, el Vicerrectorado de Asistencia al Universitario de la Universidad de Salamanca, a través del Servicio de Orientación al Universitario, detectó la existencia de problemáticas psicológicas y psicosociales para las que no se disponía de un servicio específico y articuló un proyecto al respecto.

Para crear el dispositivo, en la primavera de 1996 se firmó un acuerdo entre el Vicerrectorado de Asistencia al Universitario y el Departamento de Personalidad, Evaluación y Tratamiento Psicológicos, para la creación y desarrollo de una Unidad de atención psicológica y salud mental al universitario radicada en la Facultad de Psicología, cuyo proyecto, durante el período 1996-1999, se articuló en torno a tres subunidades: Psicología, Psiquiatría y Trabajo Social, vinculadas a las áreas de conocimiento y departamentos correspondientes. Superada la fase de organización inicial, y gracias a un excelente equipo de colaboradores desinteresados, 
se consideró consolidada la oferta a la comunidad universitaria de un servicio de calidad, dentro de sus posibilidades y límites, que continuó ofertándose y ampliándose en sus contenidos.

Hemos de destacar además, que durante esos cuatros años, y tras la notable demanda de los Universitarios hacia este servicio, nuestro interés fue crear un servicio complementario y no alternativo a los centros de la red pública asistencial, mediante una estrategia de coordinación con la Red Pública Asistencial de Salud Mental y Servicios Sociales de Salamanca. También se establecieron contactos con otras entidades y servicios de la comunidad, intentando compaginar los recursos existentes, y logrando así desarrollar un servicio de calidad, para lo cuál fue necesario no solo una adecuada coordinación con dichas redes, sino también formar adecuadamente a los colaboradores de nuestra Unidad.

El Proyecto entró entonces en una segunda fase (1999-2004), enmarcada en el Servicio de Orientación al Universitario dependiente del Vicerrectorado de Asistencia al Universitario, encargado de atender las problemáticas generales que afectan al estudiante en el marco de la convivencia - y no sólo de la formación y docencia- que constituye la Universidad. Las características específicas de dispersión de la población universitaria que se había de atender, procedente de diversas áreas de la extensa Castilla y León pero también de otras Comunidades autónomas condicionó las características de la población y el tipo de problemática que atendimos (gran parte de ella relacionada con el desarraigo, trastornos adaptativos, cuadros ansiosos, etc.).

- El fin de la Unidad fue constituir un servicio dirigido a la Comunidad universitaria, encargado de detectar los principales problemas psicológicos y de salud mental que sus miembros presenten, y tratarlos, bien a través de sus propios programas de actuación, o en coordinación con los Servicios públicos correspondientes.

> Las tareas a desarrollar por los profesionales y colaboradores de la Unidad incluyeron el Diagnóstico, orientación y tratamiento de la problemática atendida, así como la evaluación continua de las acciones realizadas.
> El marco de actuación es la intervención individual o grupal, de asistencia gratuita en los programas asistenciales.

- Las garantías de confidencialidad y respeto a los usuarios (miembros de la comunidad universitaria) se inscribieron en el marco de la deontología profesional de los profesionales implicados. Los proyectos de investigación que se articularon con el Programa se ajustaron a las exigencias de los convenios internacionales en materia de derechos humanos del sujeto que participa en investigaciones.

\section{Marco específico}

El objetivo general de la UAPSMU fue implementar y desarrollar programas de promoción, prevención y atención a las necesidades que, en materia de Atención psicológica y salud mental sean detectadas en la Comunidad universitaria, de forma coordinada con los dispositivos y redes de salud mental y servicios sociales y vinculados a los fines docentes y de investigación de la Universidad de Salamanca.

Como objetivos específicos estuvieron los siguientes:

- Detección de las necesidades de promoción, prevención y atención en Atención psicológica y salud mental de los Universitarios de Salamanca, mediante el análisis de las demandas recibidas y la realización de estudios prospectivos.

- Promoción de actitudes y comportamientos saludables mediante la participación en planes de salud.

- Prevención de desajustes, disfunciones y trastornos del comportamiento y salud mental, sobre todos de aquellos que se manifiestan más característicamente en el entorno universitario, y que pudieran estar potenciados por características del propio entorno.

- Atención a los mismos que demanden los universitarios. Para ello se desarrollarán programas de intervención propios y estrategias de derivación a los servicios especializados pertinentes. 
- Formación especializada de carácter eminentemente práctico, orientada tanto a complementar los programas docentes de segundo y tercer ciclo en materia de atención psicológica y salud mental, como a la formación de los propios colaboradores de la Unidad, mediante la implementación de Talleres, Cursos extraordinarios y otros dispositivos docentes externos al curriculum ordinario.

- Investigación, en el Marco de los programas I+D, para atender a las necesidades de la propia Unidad, y el desarrollo de técnicas e instrumentos para la implementación de programas, mediante el impulso para la realización de Tesinas y trabajos de investigación del tercer ciclo.

\section{Recursos humanos}

La UAPSMU contó, desde su inicio hasta nuestros días, con una estructura piramidal organizada con las siguientes figuras y funciones:

- Un Director: Coordina la consecución de los objetivos planteados, mediante la supervisión y planificación de los programas y puesta en marcha de las acciones de formación e investigación que se generan desde la Unidad. Así mismo, se encarga de concertar o ejecutar valoraciones externas a la misma, que posibiliten la evaluación o contraste externo.

- Un Subdirector: Encargado de las funciones de dirección en ausencia de éste, así como de trasladar las propuestas de los distintos responsables de los subprogramas a la Dirección y posibilitar la implementación de nuevas propuestas dentro de la Unidad.

- Un coordinador clínico: Coordina los Subprogramas de recepción de demandas y de Derivación, así como el de Diagnóstico y valoración psicológica, encargándose así mismo de la articulación entre los diferentes subprogramas de tratamiento.

- Un responsable por cada uno de los Subprogramas: Encargados de la puesta en marcha, ejecución, y supervisión de los mismos.

- Dos becarios colaboradores: Encargados de funciones administrativas y de apoyo.

Las colaboraciones se rigieron por el Reglamento de colaboraciones que se recoge como Anexo I.

\section{Subprogramas de funcionamiento}

- De Recepción de demanda y derivaciones:

La Unidad de Información del SOU, los PIU (Puntos de Información Universitaria), los propios programas de la UAPSMU, la Unidad de Trabajo Social, la Unidad de Psiquiatría y Psicología Médica, los dispositivos de la red pública de Salud y Servicios Sociales, todos ellos actuaban de puerta de entrada a los servicios de la UAPSMU, además del cada vez más significativo volumen de demandas recibido directamente desde los propios miembros de la Comunidad Universitaria. El acceso al Programa de Recepción de demandas y derivación se hacía a través del sistema de cita previa, en el que la ficha de datos que se cumplimenta bien telefónica o personalmente facilitando así las acciones de recepción.

\section{- Diagnóstico y valoración psicológica:}

El Programa de Diagnóstico y Valoración psicológica cumple dos finalidades principales: a) llevar a cabo las valoraciones psicológicas y emisión de informes para que el servicio de recepción de demandas y derivación pueda desarrollar adecuadamente su labor; b) facilitar informes específicos para que sea aprovechada en la planificación de las intervenciones a realizar en los diferentes subprogramas de atención y tratamiento.

- Subprograma de Trastornos del Comportamiento.

Las temáticas, sobre las que puede focalizarse la intervención, son muy variadas. En el primer período de intervención se inició un protocolo de intervención en Fo- 
bias simples. En este subprograma se ofertó intervención individualizada para el tratamiento de aquellas fobias simples que interfieran con el funcionamiento adaptativo del alumno. Por tratarse de fobias simples, en este caso ofertamos como método de intervención la Desensibilización Sistemática, dado que su duración es breve y altamente eficaz. Si se diese el caso de que varios alumnos presentasen el mismo tema fóbico, se realiza el tratamiento a nivel grupal. La duración media de estas intervenciones es de ocho a diez sesiones. Pero en cada caso, variaría en función de la problemática a tratar.

- De sensibilización, comunicaciones y habilidades interpersonales.

Una buena parte de las demandas se refieren a la gestión de necesidades vitales, crisis de crecimiento y adaptación, comunes a la problemática del sector de población dominante en el entorno universitario y especialmente precipitados por la natural situación de desarraigo familiar que atraviesan una proporción importante de los alumnos de la Universidad de Salamanca. Intervenciones individuales de corta duración, gestionadas con técnicas de apoyo focal, combinadas con técnicas de grupo, dan un excelente rendimiento en el afrontamiento de estas crisis, sin que se psicologice el problema, recibiendo ayuda en su medio natural, la universidad. El dispositivo de intervención individual no excede de las 20 sesiones, con un promedio de intervención por caso de 12 sesiones. El dispositivo de grupo es de periodicidad semanal, durante el periodo lectivo, normalmente no excede de 25-30 sesiones.

\section{- De atención neuropsicológica:}

Una proporción significativa de sujetos que han sufrido un Traumatismo CraneoEncefálico (T.C.E.), a pesar de presentar una buena resolución neurológica después del accidente, manifiestan déficits cognitivos-comportamentales (fundamentalmente atencionales, memoria, lenguaje). Estos déficit pueden afectar de forma significativa a la integración social, familiar y aca- démica del sujeto. La evolución-recuperación de dichos déficit se va a ver influida por factores de diversa índole. Desde factores puramente biográficos del sujeto (personalidad premórbida, nivel educacional) y factores neuro-médicos relacionados con el T.C.E. (Intensidad-severidad del T.C.E., tipo de daño neurológico: focal/ difuso, tipo específico de daño neuropsicológico, existencia o no de complicaciones neurológicas y neuroquirúrgicas).

- De Ansiedad ante los exámenes y Autocontrol para el estudio:

Es común que lleguen a los servicios de orientación universitaria estudiantes con problemas para estudiar. Los factores responsables pueden ser de diversa índole, entre los que podríamos citar, entre otros, tener metas a largo plazo poco realistas, una seria falta de preparación escolar, etc. Sin embargo, la dificultad general está en función de hábitos de estudio ineficaces y del tiempo insuficiente que se dedica al mismo. El programa de ansiedad ante los exámenes intenta proporcionar a los alumnos que padezcan dicho trastorno los medios adecuados para superarla. El programa de autocontrol va dirigido a aquellos alumnos de los que, tras una cuidadosa evaluación, puede decirse que la causa principal de su pobre rendimiento académico es una ineficaz programación de tiempo que dedican al estudio.

\section{Análisis de la demanda}

Las tablas y gráficos que siguen recogen los principales indicadores del funcionamiento de la UAPSMU desde su creación en 1996 hasta la reformulación de su proyecto en 2004. Los datos están separados por cursos académicos, y debe tener se en cuenta que en el primer período (1996-97) solo se pudo computar el segundo cuatrimestre (el servició se articuló al principio de la primavera, si bien la importante demanda inicial acumulada hizo que fuera un período de intensidad equivalente a los otros. También que las estadísticas sistemáticas solo incluyen hasta el final del período 2002-2003, ya que las es- 
tructuras de funcionamiento sufrieron un importante cambio al inicio de la temporada 20032004, debido a un cambio en las directrices emanadas del equipo rectoral en activo ese momento. El periodo que describen las tablas 3 a 7 y sus gráficos ilustrativos se corresponden pues con la etapa académica 1996-2003, siete cursos completos, en los que la UAPSMU funcionó de manera efectiva de acuerdo a los programas que se describen en este trabajo.

Los principales indicadores recogidos en la tabla 2 muestran que la UAPSMU recibió un importante volumen de demandas (en el rango 128-199 consultantes por año), tendente al alza, que fue parejo con la prestación de una asistencia más continuada (La media de consultas por caso evoluciona de 5 a 16,1), dentro del carácter de intervención focal-breve que tiene este esquema asistencial, y remarcando también el drástico recorte del número de consultas por usuario en el último año, debido a la pérdida de recursos humanos por el cambio de política rectoral sobre la dotación de medios para esta unidad. La tabla 3 subraya el predominio de la demanda de mujeres $(>70 \%)$, si bien se observa una tendencia progresiva a reducir este sesgo de género en la demanda. La tabla 4 muestra que el grupo de edad más característico está en el corte 20-23 años, congruente no solo con las medias de edad de la población universitaria, sino también con la evidencia de que la demanda de ayuda no se formula según se ingresa en la universidad, sino al menos tras uno o dos años de permanencia en la misma, cuando ya se tiene una percepción más clara de las necesidades (p.e. tras los primeros fracasos crisis), y también de los recursos que pueden usarse. La tabla 5 nos muestra que los principales usuarios provienen de los propios estudios de Psicología, así como de otros estudios de Humanidades y Ciencias Sociales, tendencia que puede

Tabla 2. Comunidad universitaria demandante de atención psicológica.

\begin{tabular}{|l|c|c|c|c|c|c|c|}
\hline & $\mathbf{1 9 9 6 - 9 7}$ & $\mathbf{1 9 9 7 - 9 8}$ & $\mathbf{1 9 9 8 - 9 9}$ & $\mathbf{1 9 9 9 - 0 0}$ & $\mathbf{2 0 0 0 - 0 1}$ & $\mathbf{2 0 0 1 - 0 2}$ & $\mathbf{2 0 0 2 - 0 3}$ \\
\hline DA & 128 & 140 & 199 & 131 & 141 & 143 & 189 \\
\hline NC & 560 & 868 & 2985 & 2001 & 2185 & 2302 & 1532 \\
\hline MC & 5 & 6,2 & 15,1 & 14,5 & 15,5 & 16,1 & 5,1 \\
\hline
\end{tabular}

Nota: DA (Demandas de Atención); NC (Número de consultas); MC (Media consultas).

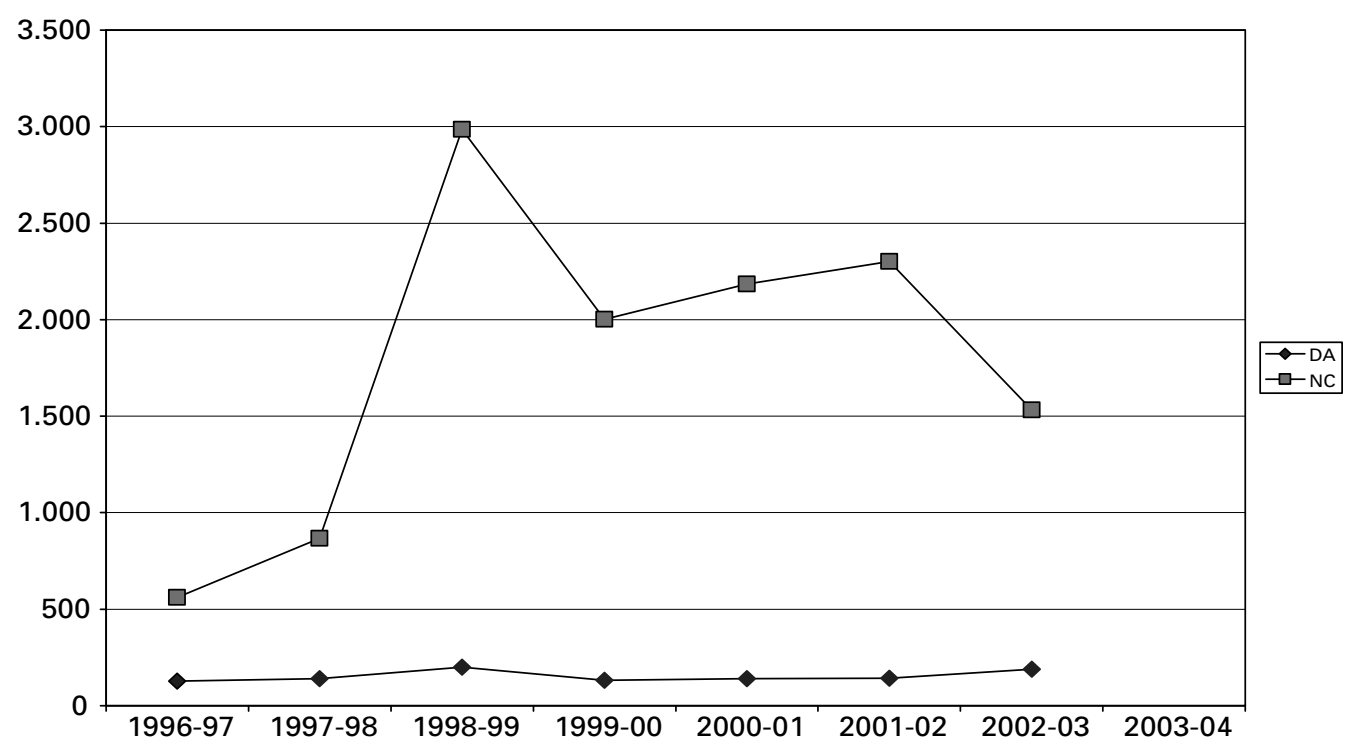


Tabla 3. Distribución por género de la población atendida (en porcentaje).

\begin{tabular}{|l|c|c|c|c|c|c|c|c|}
\hline & $\mathbf{1 9 9 6 - 9 7}$ & $\mathbf{1 9 9 7 - 9 8}$ & $\mathbf{1 9 9 8 - 9 9}$ & $\mathbf{1 9 9 9 - 0 0}$ & $\mathbf{2 0 0 0 - 0 1}$ & $\mathbf{2 0 0 1 - 0 2}$ & $\mathbf{2 0 0 2 - 0 3}$ & $\mathbf{2 0 0 3 - 0 4}$ \\
\hline Mujeres & 84 & 79 & 82 & 80 & 73 & 73 & 72 & \\
\hline Hombres & 16 & 21 & 18 & 20 & 27 & 27 & 28 & \\
\hline
\end{tabular}

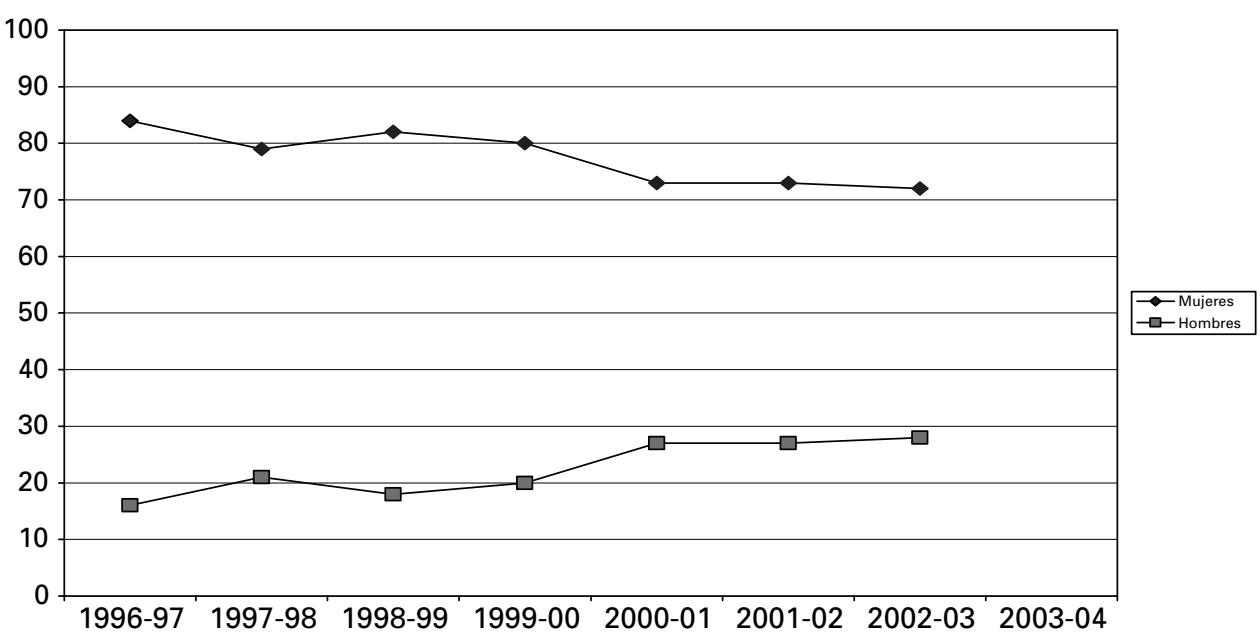

Tabla 4. Distribución por edades (en porcentaje).

\begin{tabular}{|c|c|c|c|c|c|c|c|}
\hline & $\mathbf{1 9 9 6 - 9 7}$ & $\mathbf{1 9 9 7 - 9 8}$ & $\mathbf{1 9 9 8 - 9 9}$ & $\mathbf{1 9 9 9 - 0 0}$ & $\mathbf{2 0 0 0 - 0 1}$ & $\mathbf{2 0 0 1 - 0 2}$ & $\mathbf{2 0 0 2 - 0 3}$ \\
\hline$<20$ & 33 & 13 & 2 & 3 & 9 & 8 & 18 \\
\hline $20-23$ & 39 & 47 & 66 & 61 & 54 & 52 & 58 \\
\hline $24-27$ & 21 & 29 & 22 & 25 & 25 & 30 & 13 \\
\hline$>27$ & 7 & 11 & 10 & 11 & 12 & 10 & 11 \\
\hline
\end{tabular}

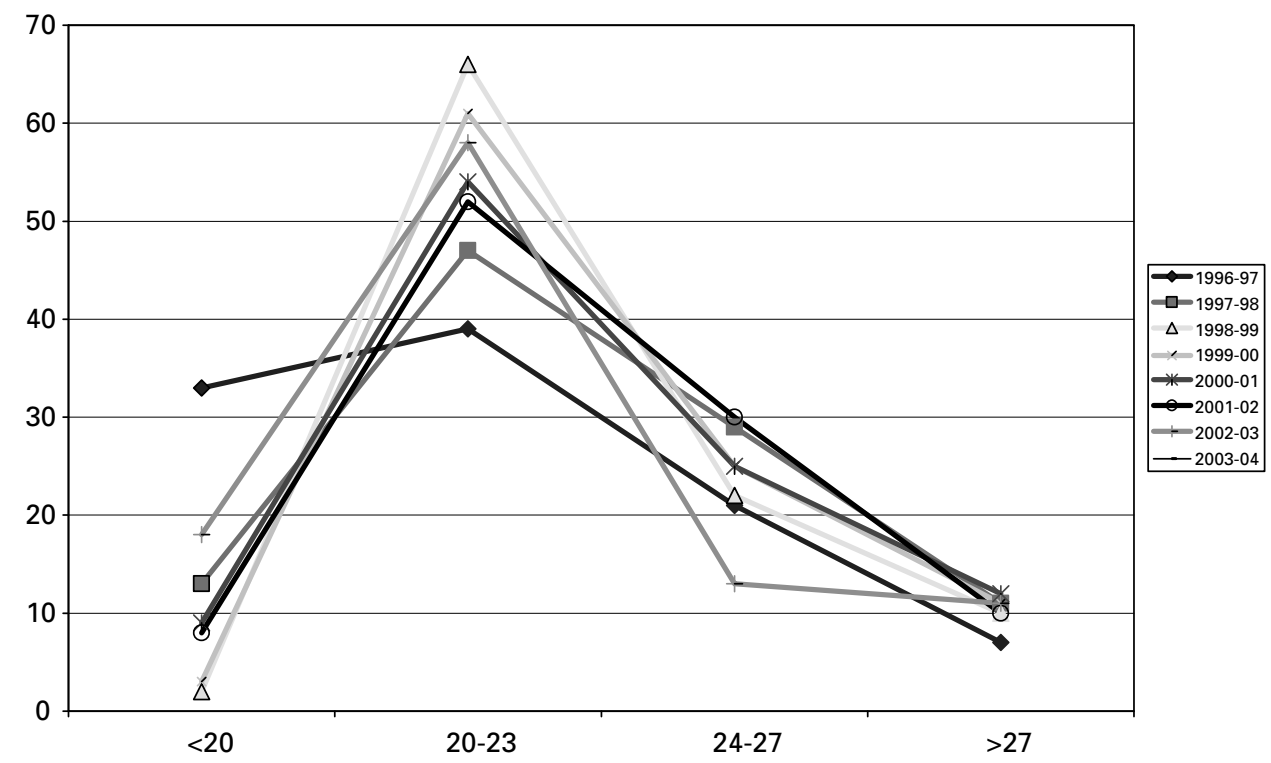


Tabla 5. Distribución por estudios cursados (en porcentaje).

\begin{tabular}{|l|c|c|c|c|c|c|c|}
\hline & $\mathbf{1 9 9 6 - 9 7}$ & $\mathbf{1 9 9 7 - 9 8}$ & $\mathbf{1 9 9 8 - 9 9}$ & $\mathbf{1 9 9 9 - 0 0}$ & $\mathbf{2 0 0 0 - 0 1}$ & $\mathbf{2 0 0 1 - 0 2}$ & $\mathbf{2 0 0 2 - 0 3}$ \\
\hline P & 40 & 35 & 25 & 21 & 26 & 18 & 18 \\
\hline CS & 6 & 15 & 12 & 8 & 7 & 10 & 12 \\
\hline HCS & 10 & 12 & 53 & 53 & 35 & 40 & 41 \\
\hline CT & 5 & 10 & 8 & 12 & 6 & 15 & 5 \\
\hline PCE & 0 & 0 & 0 & 6 & 6 & 0 & 0 \\
\hline OC & 0 & 0 & 0 & 0 & 20 & 17 & 24 \\
\hline NC & 39 & 28 & 2 & 0 & 0 & 0 & 0 \\
\hline
\end{tabular}

Nota: P (Psicología); CS (Ciencias de la Salud); HCS (Humanidades y Ciencias Sociales); CT (Carreras Técnicas); PCE (Postgrado y Cursos Extraordinarios); OC (Otras carreras de Ciencias); NC (No Contesta).

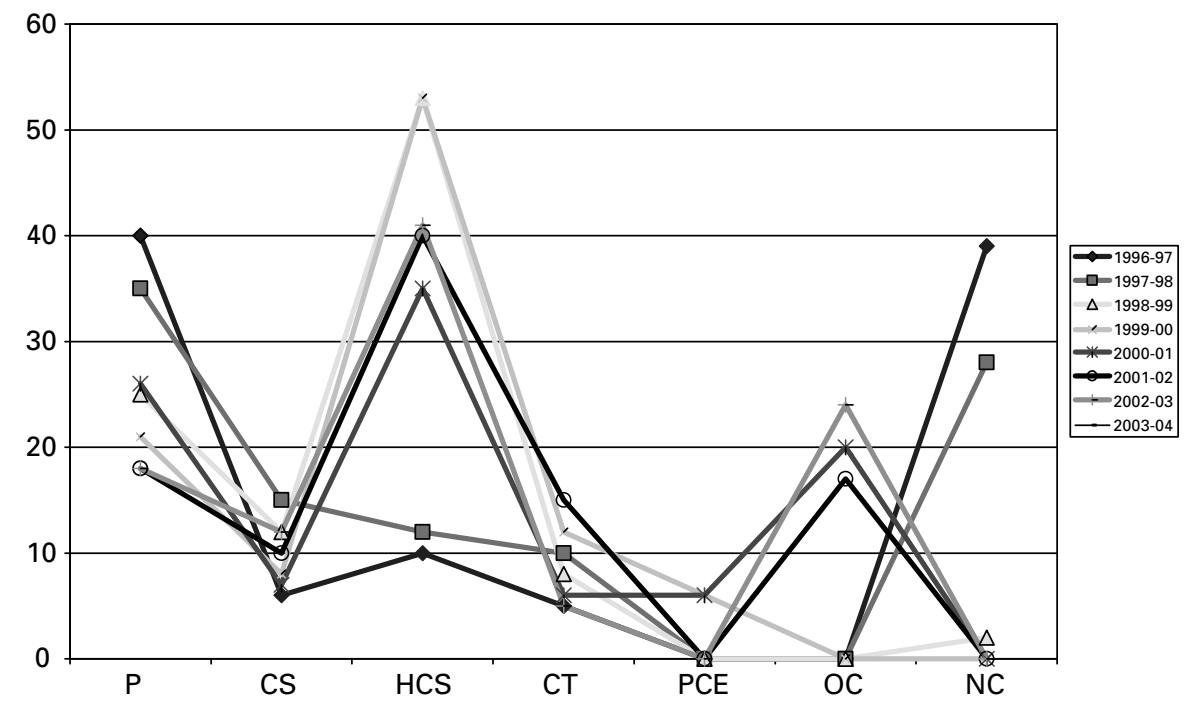

deberse tanto a una mayor percepción de la propia conflictividad en estos grupos, como por su mayor cercanía y conocimiento de los servicios. Por ello dirigimos también nuestros esfuerzos a dar a conocer los programas de la Unidad entre las carreras de Ciencias y Salud. La tabla 6 muestra como los principales usuarios son los que proceden de otras áreas geográficas distintas de Salamanca, y conecta con la relevancia de los programas específicos de atención a la problemática del desarraigo. Finalmente la tabla 7 destaca los cuatro grandes temas dominantes en las demandas del último período estudiado (2002-2003), pero que es re- presentativo de lo observado en los períodos anteriores. Los trastornos de ansiedad, estado de ánimo, desadaptación-baja autoestima-déficit de habilidades sociales, y finalmente las cuadros de ansiedad ante los exámenes, son los ejes que motivan la demanda, y que deben configurar el esquema de intervención preferente en este tipo de unidades. Sin que le demos mayor relevancia a esos datos, los indicadores de satisfacción de los usuarios con el servicio, mediante cuestionarios anónimos a la terminación o interrupción de la intervención, siempre estuvieron por encima del $90 \%$ de usuarios satisfechos y muy satisfechos. 
Tabla 6. Distribución por procedencia geográfica (en porcentaje).

\begin{tabular}{|l|c|c|c|c|c|c|c|}
\hline & $\mathbf{1 9 9 6 - 9 7}$ & $\mathbf{1 9 9 7 - 9 8}$ & $\mathbf{1 9 9 8 - 9 9}$ & $\mathbf{1 9 9 9 - 0 0}$ & $\mathbf{2 0 0 0 - 0 1}$ & $\mathbf{2 0 0 1 - 0 2}$ & $\mathbf{2 0 0 2 - 0 3}$ \\
\hline SA & 18 & 26 & 22 & 25 & 21 & 23 & 21 \\
\hline RE & 82 & 74 & 71 & 68 & 70 & 70 & 55 \\
\hline EX & 0 & 0 & 7 & 7 & 9 & 7 & 24 \\
\hline
\end{tabular}

Nota: SA (Salamanca); RE (Resto de España); EX (Extranjeros).

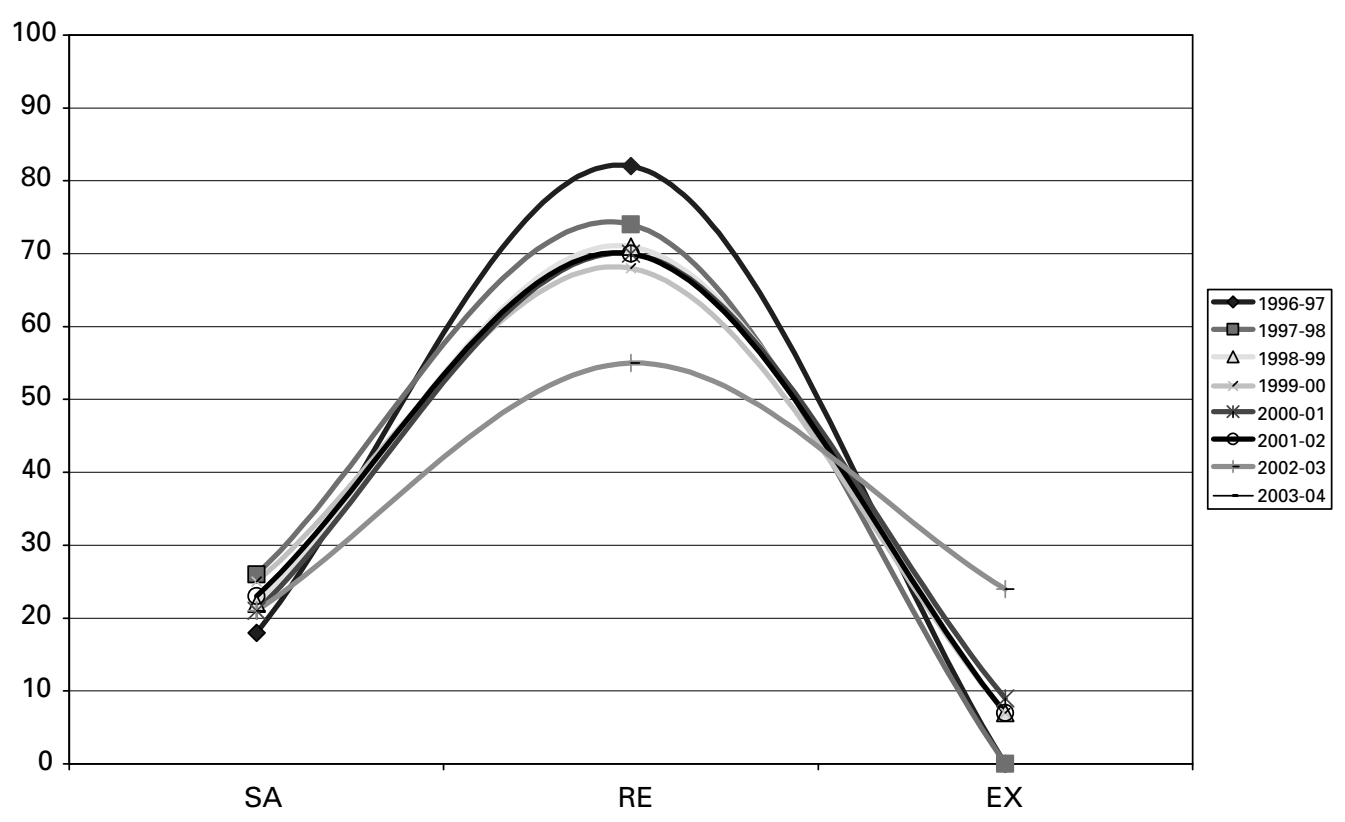

Tabla 7. Tipo de demandas recibidas en la UAPSMU (Curso 2002/2003).

\begin{tabular}{|l|c|}
\hline \multicolumn{1}{|c|}{ Demandas: categoría diagnóstica } & Frecuencia \\
\hline Trastorno de Ansiedad & 62 \\
\hline Trastorno del Estado de Ánimo & 45 \\
\hline Trastorno Adaptativo/Habilidades Sociales/Baja Autoestima & 27 \\
\hline Problemas Control Estudio/Ansiedad ante los Exámenes & 26 \\
\hline Trastorno de Alimentación & 11 \\
\hline Problemas de Relación Interpersonal & 6 \\
\hline Problemas en el Control de Impulsos & 5 \\
\hline Trastorno de Personalidad (con relevancia diagnóstica) & 3 \\
\hline Peticiones de Orientación/Información & 3 \\
\hline Duelo Patológico & 1 \\
\hline Abuso de Sustancias & 0 \\
\hline
\end{tabular}




\section{Conclusiones}

Haber participado en el desarrollo de un proyecto como la UAPSMU confiere un sentido diferente al trabajo universitario. Todos los que tuvimos esta excepcional posibilidad pudimos vivir la trascendencia humana e institucional de esta experiencia, donde más allá de los esquemas y planteamientos docentes y de investigación, los profesores estables, los becarios, nuestros alumnos de postgrado, y los usuarios del servicio compartíamos una tarea donde se alcanzaba el sentido integral de la palabra Universidad. El estudio y el descubrimiento no solo no son incompatibles con el desarrollo de la subjetividad, sino que dan el sentido al recorrido humano que todos hacemos en la universidad, cada uno desde nuestras posiciones y medios. Algunas evidencias fueron surgiendo en el desarrollo de nuestra tarea, como ejes naturales para lograr un servicio público de calidad: la importancia decisiva del apoyo institucional de la Universidad, ilusionado con el proyecto, dotándolo de los medios y recursos necesarios. La gratuidad, tanto de las colaboraciones como de los servicios prestados, sin perjuicio de reconocer a los profesores su dedicación para no aumentar su sobrecarga. Y finalmente el carácter «cerrado» de la prestación, es decir que se oferte solo a los miembros de la comunidad universitaria, para evitar «competir» con servicios públicos o privados, y bajo un esquema de intervención focalizada en los objetivos, y limitada en el tiempo, coordinada con las restantes redes asistenciales que la comunidad ofrece. Habrá otros modelos relevantes y valiosos, pero éste que proponemos, en base a nuestra experiencia, nos parece el más adecuado a los ideales de la Universidad pública.

Y por haber tenido esta oportunidad, y en el nombre de quienes nos acompañaron en ese recorrido, así lo agradecemos.

\section{Referencias}

Barrio, V. y Carpintero, H. (2003). Clinical Psychology in Spain. Journal of Clinical Psychology, 59(6), 687-699.

UAPSMU (1997). Memoria 1996-1997 de la Unidad de Atención Psicológica. Memorias Anuales de la
Unidad de Atención Psicológica y Salud Mental al Universitario. Manuscrito no publicado, Universidad de Salamanca, España.

UAPSMU (1998). Memoria 1997-1998 de la Unidad de Atención Psicológica. Memorias Anuales de la Unidad de Atención Psicológica y Salud Mental al Universitario. Manuscrito no publicado, Universidad de Salamanca, España.

UAPSMU (1999). Memoria 1998-1999 de la Unidad de Atención Psicológica. Memorias Anuales de la Unidad de Atención Psicológica y Salud Mental al Universitario. Manuscrito no publicado, Universidad de Salamanca, España.

UAPSMU (2000). Memoria 1999-2000 de la Unidad de Atención Psicológica. Memorias Anuales de la Unidad de Atención Psicológica y Salud Mental al Universitario. Manuscrito no publicado, Universidad de Salamanca, España.

UAPSMU (2001). Memoria 2000-2001 de la Unidad de Atención Psicológica. Memorias Anuales de la Unidad de Atención Psicológica y Salud Mental al Universitario. Manuscrito no publicado, Universidad de Salamanca, España.

UAPSMU (2002). Memoria 2001-2002 de la Unidad de Atención Psicológica. Memorias Anuales de la Unidad de Atención Psicológica y Salud Mental al Universitario. Manuscrito no publicado, Universidad de Salamanca, España.

UAPSMU (2003). Memoria 2002-2003 de la Unidad de Atención Psicológica. Memorias Anuales de la Unidad de Atención Psicológica y Salud Mental al Universitario. Manuscrito no publicado, Universidad de Salamanca, España.

UAPSMU (2004). Memoria 2003-2004 de la Unidad de Atención Psicológica. Memorias Anuales de la Unidad de Atención Psicológica y Salud Mental al Universitario. Manuscrito no publicado, Universidad de Salamanca, España.

\section{ANEXO I:}

\section{REGLAMENTO DE COLABORACIONES CON LA U.A.P.S.M.U.}

La Unidad de Atención Psicológica y Salud Mental para el Universitario, dependiente del Servicio de Orientación al Universitario de la Universidad de Salamanca se rige por su Programa Marco y este Reglamento de Colaboración. 


\section{Tipos de Colaboración:}

Existen diferentes tipos de colaboración con la UAPSMU. Todos los participantes en la Unidad habrán de estar incluidos en alguno de ellos:

> Profesores de la Universidad de Salamanca, que colaboran voluntariamente en las distintas funciones que se están desarrollando en la Unidad, bien a nivel de primeras entrevistas, evaluación, diagnóstico y tratamiento, y/o supervisando a otros colaboradores de la Unidad.

> Becarios de Colaboración: Que además de las funciones propias estipuladas en su Beca formarán parte del equipo de la Unidad, según su nivel de formación, en las mismas condiciones que : a) los profesores; b) los colaboradores voluntarios, alumnos de tercer ciclo.

> Colaboradores voluntarios, alumnos de Tercer ciclo, en su primer año de colaboración: tras una selección de méritos y C.V., los alumnos que lo solicitan, acceden al primer año de formación en la Unidad.

> Colaboradores voluntarios alumnos de Tercer ciclo, en su segundo año de colaboración, que seguirán prestando atención psicológica en los casos y programas asignados, bajo la supervisión de otros profesionales.

- Colaboradores voluntarios alumnos de Tercer ciclo, que tras dos años de colaboración, o bien tras la extinción de la beca colaboración, quieren continuar desarrollando funciones en la Unidad, y a los que previa propuesta específica, se les autoriza temporalmente.

> Profesores Visitantes de otras Universidades, ya sean Españolas o Extranjeras, previa autorización del Director de la Unidad.

- Alumnos de la Licenciatura en Psicología, cursando el Practicum de la misma: alumnos de psicología en su quinto año de carrera. Aceptan un compromiso específico de acuerdo a su nivel de participación.

\section{Duración de la colaboración:}

- Profesores de la Universidad de Salamanca: Ilimitada.

> Becarios de Colaboración: Hasta dos años.
> Colaboradores voluntarios, alumnos de Tercer ciclo: Hasta dos años, con posible prórroga previa propuesta..

- Profesores Visitantes de otras Universidades: La duración de su visita.

- Alumnos de la Licenciatura en Psicología, cursando el Practicum de la misma: 90 horas en un año académico.

\section{Compromisos aceptados por los Profe- sores:}

1. Prestar a la Unidad una dedicación personal en carácter de voluntariado durante el período académico (Periodos de 1 de Septiembre a 30 de Julio). Asumo la realización de hasta 2 primeras entrevistas al mes, y la atención e intervención con hasta dos casos simultáneos. Además me comprometo a colaborar en el Programa formativo que oferta la Unidad y en la Supervisión de los colaboradores.

2. Si bien tengo la libertad de abandonar la colaboración en cualquier momento, acepto el compromiso moral de continuidad y dedicación durante al menos dos años. Mi colaboración es voluntaria y no supone modificación de mi vínculo laboral con la Universidad, ni contraprestación económica.

3. Conocer y guardar las garantías deontológicas profesionales que me obligan, entre otras, a la absoluta confidencialidad y respeto de los derechos de los consultantes que acuden a la U.AP.S.M.U. Como norma general, en las comunicaciones internas referidas a los consultantes mencionaré el código identificador y nunca el nombre del consultante.

4. Establecer y respetar un compromiso de asiduidad, continuidad y puntualidad en el trabajo con los consultantes: Asistiré a todas las citas concertadas con el consultante, y avisaré con antelación suficiente a la secretaría de la Unidad de cualquier modificación (48 horas de antelación, en días de funcionamiento de la Unidad).

5. Realizaré los informes pertinentes respecto de las intervenciones realizadas.. Dichos informe los entregaré en la Unidad en sobre cerrado marcado con su código identifica- 
dor, en los diferentes momentos en que sea necesario: Derivación; Asignación de Tratamiento; Alta; Abandono.

6. Accederé a la consulta de los protocolos, guías y esquemas de trabajo de la Unidad. Podré utilizar material (Libros, protocolos, técnicas de evaluación, otra información) existente en la Unidad, mediante un servicio de préstamo. Cuidaré de este material, que habré de devolver en perfecto estado. En la medida de mis posibilidades contribuiré a la mejora de dichos protocolos, o bien desarrollaré otros nuevos, sí así se me pide, que quedarán en la Unidad como material de trabajo.

7. Utilizaré con discreción y racionalidad al material fungible que se me facilite, y para usos propios de la Unidad.

8. Utilizaré los locales de la Unidad que se me asignen para realizar sólo y exclusivamente tareas propias de la Unidad, respetando los turnos y horarios asignados por secretaría.

9. Si existieran circunstancias especiales por las cuáles no pueda hacerse cargo de algún caso deberé de manifestarlo lo antes posible para que sea asignado a otro colaborador.

10. Ajustar mi actividad a las normas de registro y protocolización de la Unidad (hojas de actividad diaria, formularios, etc.), siguiendo las indicaciones y pautas que se me faciliten.

11. Participaré, en la medida de lo posible, en las actividades comunes que organice la Unidad: Sesiones clínicas, conferencias, reuniones de equipo, etc. En todo caso mantendré la coordinación que sea necesaria para garantizar el buen funcionamiento y atención a los casos.

\section{Compromisos aceptados por los colabo- radores voluntarios alumnos de tercer ciclo.}

1. Prestar a la Unidad una dedicación personal en carácter de voluntariado, de dos años de duración (Periodos de 15 de Septiembre a 15 de Julio). El primer año un mínimo de ocho horas semanales de presencia en las actividades de la Unidad, más el tiempo personal de preparación y elaboración ne- cesario. En el segundo año, de un mínimo de seis horas semanales de presencia en las actividades de la Unidad, más el tiempo personal necesario. Si bien tengo la libertad de abandonar la colaboración en cualquier momento, acepto el compromiso moral de continuidad y dedicación antes expresado. Mi colaboración es voluntaria y no supone actividad o vinculo laboral ni contraprestación económica. Acepto como suficiente y satisfactoria contraprestación a mi colaboración la formación y supervisión gratuita que oferta la Unidad.

2. Conocer y guardar las garantías deontológicas profesionales que me obligan, entre otras, a la absoluta confidencialidad y respeto de los derechos de los consultantes que acuden a la U.AP.S.M.U. Como norma general, en las comunicaciones internas referidas a los consultantes mencionaré el código identificador y nunca el nombre del consultante.

3. Desarrollar las tareas clínicas que se me asignen, principalmente las de: evaluación, diagnóstico, orientación y tratamiento psicológicos, bajo las oportunas supervisiones.

4. Establecer y respetar un compromiso de asiduidad, continuidad y puntualidad en el trabajo con los consultantes: Asistiré a todas las citas concertadas con el consultante, y avisaré con antelación suficiente a la secretaría de la Unidad de cualquier modificación (48 horas de antelación, en días de funcionamiento de la Unidad).

5. Respecto de los consultantes asignados para Evaluación Psicológica Clínica, seguiré el protocolo de técnicas de evaluación establecido en la Unidad, y consultar previamente la inclusión de cualquier prueba adicional. Así mismo realizaré un informe psicológico que incluya los resultados de la evaluación, diagnóstico y/o de intervención efectuada con cada consultante. Dicho informe lo entregaré en la Unidad en sobre cerrado marcado con su código identificador, en los diferentes momentos en que sea necesario: Derivación; Asignación de Tratamiento; Alta; Abandono.

6. Utilizar los diferentes espacios de supervisión periódica que se ofertan en la Unidad: 
Supervisión del proceso de evaluación e informe; Supervisión de la derivación o asignación a tratamiento; Supervisión específica y continuada de cada caso en el que intervenga o desarrolle tratamiento.

7. Accederé a la consulta de los protocolos, guías y esquemas de trabajo de la Unidad. Podré utilizar material (Libros, protocolos, técnicas de evaluación, otra información) existente en la Unidad, mediante un servicio de préstamo. Cuidaré de este material, que habré de devolver en perfecto estado. En la medida de mis posibilidades contribuiré a la mejora de dichos protocolos, o bien desarrollaré otros nuevos, sí así se me pide, que quedarán en la Unidad como material de trabajo.

8. Utilizaré con discreción y racionalidad al material fungible que se me facilite, y para usos propios de la Unidad.

9. Utilizaré los locales de la Unidad que se me asignen para realizar sólo y exclusivamente tareas propias de la Unidad, respetando los turnos y horarios asignados por secretaría.

10. Si existieran circunstancias especiales por las cuáles usted no puede hacerse cargo de algún caso usted deberá de manifestarlo lo antes posible para que sea asignado a otro colaborador.

11. Ajustar mi actividad a las normas de registro y protocolización de la Unidad (hojas de actividad diaria, formularios, etc.), siguiendo las indicaciones y pautas que se me faciliten.

12. Conozco y acepto que si incumplo estas normas, podré ser excluido de la colaboración voluntaria con la Unidad.

\section{Compromisos aceptados por los alumnos de Practicum}

1. Guardar fielmente la información obtenida durante su periodo do de prácticas en este centro/unidad.

2. Cumplir el secreto profesional según el Código deontológico de la profesión de psicólogo.

3. Y aceptar las normas de funcionamiento del centro/unidad y sus responsables

\section{Contraprestaciones que ofrece la Unidad}

\section{Para los profesores:}

1. Facilitar el acceso gratuito a las actividades de formación e intercambio de experiencias en Psicología Clínica profesional que promueva esta Unidad al efecto.

2. Pondrá a su disposición el material de trabajo de la Unidad cuando lo precise, ya sean libros, protocolos, pruebas diagnósticas, etc. También tendrá a su disposición un despacho compartido con los demás miembros de la Unidad para la atención a los consultantes.

3. Promoverá la adquisición de experiencia clínica, dentro de los programas propios de la Unidad, tanto a nivel de evaluación como de intervención.

\section{Para los colaboradores voluntarios:}

1. Facilitar el acceso gratuito a las actividades de formación en Psicología Clínica profesional que promueva esta Unidad al efecto.

2. Esta formación comprenderá al menos seminarios teórico-prácticos, sesiones clínicas, y conferencias durante un mínimo de 60 horas anuales.

3. Facilitar la supervisión de las distintas tareas que asuma a nivel clínico, tanto mediante supervisiones en grupo, como individuales, cuando proceda.

4. Facilitar el aprendizaje práctico de una variedad de técnicas de evaluación y diagnóstico clínico, así como de los informes psicológicos.

5. Pondrá a su disposición el material de trabajo de la Unidad cuando lo precise, ya sean libros, protocolos, pruebas diagnósticas, etc. También tendrá a su disposición un despacho compartido con los demás miembros de la Unidad para la atención a los consultantes.

6. Promover la adquisición de experiencia clínica, dentro de los programas propios de la Unidad, tanto a nivel de evaluación como de intervención. 